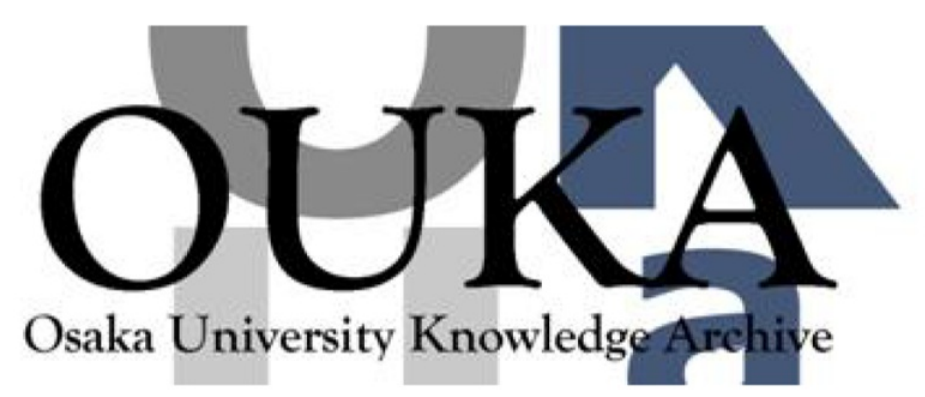

\begin{tabular}{|c|c|}
\hline Title & $\begin{array}{l}\text { Quantum Valence Criticality as an Origin of } \\
\text { Unconventional Critical Phenomena }\end{array}$ \\
\hline Author(s) & Watanabe, Shinji; Miyake, Kazumasa \\
\hline Citation & $\begin{array}{l}\text { Physical Review Letters. 105(18) p. 186403_1- } \\
\text { p. } 18640344\end{array}$ \\
\hline Issue Date & $2010-10-29$ \\
\hline oaire:version & VoR \\
\hline URL & https://hdl. handle. net/11094/2994 \\
\hline rights & $\begin{array}{l}\text { Watanabe, Shinji, Miyake, Kazumasa, Physical } \\
\text { Review Letters, 105, 18, 186403, 2010-10-29. } \\
\text { "Copyright } 2010 \text { by the American Physical } \\
\text { Society." }\end{array}$ \\
\hline Note & \\
\hline
\end{tabular}

Osaka University Knowledge Archive : OUKA

https://ir. Library. osaka-u. ac. jp/

0saka University 


\title{
Quantum Valence Criticality as an Origin of Unconventional Critical Phenomena
}

\author{
Shinji Watanabe and Kazumasa Miyake \\ Division of Materials Physics, Department of Materials Engineering Science, Graduate School of Engineering Science, \\ Osaka University, Toyonaka, Osaka 560-8531, Japan \\ (Received 6 April 2010; published 28 October 2010)
}

\begin{abstract}
It is shown that unconventional critical phenomena commonly observed in paramagnetic metals $\mathrm{YbRh}_{2} \mathrm{Si}_{2}, \mathrm{YbRh}_{2}\left(\mathrm{Si}_{0.95} \mathrm{Ge}_{0.05}\right)_{2}$, and $\beta$ - $\mathrm{YbAlB}_{4}$ are naturally explained by the quantum criticality of $\mathrm{Yb}$-valence fluctuations. We construct the mode-coupling theory taking account of local correlation effects of $f$ electrons and find that unconventional criticality is caused by the locality of the valence fluctuation mode. We show that measured low-temperature anomalies such as divergence of uniform spin susceptibility $\chi \sim T^{-\zeta}$ with $\zeta \sim 0.6$ giving rise to a huge enhancement of the Wilson ratio and the emergence of $T$-linear resistivity are explained in a unified way.
\end{abstract}

DOI: 10.1103/PhysRevLett.105.186403

PACS numbers: 71.27.+a, 71.10.-w, 71.20.Eh, 75.20.Hr

Anomalous critical phenomena, which do not follow the conventional spin-fluctuation theories [1-4] have been discovered in paramagnetic metal phases in $\mathrm{YbRh}_{2} \mathrm{Si}_{2}[5,6]$, $\mathrm{YbRh}_{2}\left(\mathrm{Si}_{0.95} \mathrm{Ge}_{0.05}\right)_{2}$ [7,8], and $\beta$ - $\mathrm{YbAlB}_{4}$ [9]. The most striking anomaly is that low-temperature uniform spin susceptibility exhibits divergent behavior $\chi(T) \sim T^{-\zeta}$ with the anomalous critical exponent $\zeta=0.6$ in $\mathrm{YbRh}_{2}\left(\mathrm{Si}_{0.95} \mathrm{Ge}_{0.05}\right)_{2}$ and $\zeta=0.5$ in $\beta$ - $\mathrm{YbAlB}_{4}$ in spite of no sign of a ferromagnetic phase nearby. In these materials, the Sommerfeld constant exhibits the logarithmic divergence $\gamma_{e}=C_{e} / T \sim-\ln T[5,8,9]$, giving rise to a large Wilson ratio, i.e., a dimensionless ratio of $\chi$ to $\gamma_{e}, R_{W}=17.5$ in $\mathrm{YbRh}_{2}\left(\mathrm{Si}_{0.95} \mathrm{Ge}_{0.05}\right)_{2}$ [7], and $R_{W}=6.5$ in $\beta$ - $\mathrm{YbAlB}_{4}$ [9], exceeding the conventional strongcoupling value $R_{W}=2$. The linear- $T$ dependence of low- $T$ resistivity emerges in a wide- $T$ range in $\mathrm{YbRh}_{2} \mathrm{Si}_{2}$ [5], $\mathrm{YbRh}_{2}\left(\mathrm{Si}_{0.95} \mathrm{Ge}_{0.05}\right)_{2}$ [8], and also $\beta$ - $\mathrm{YbAlB}_{4}$ [9]. These observations suggest that there exists a new class of materials showing similar critical phenomena, which are unconventional.

So far, to explain $\mathrm{YbRh}_{2} \mathrm{Si}_{2}$ and $\mathrm{YbRh}_{2}\left(\mathrm{Si}_{0.95} \mathrm{Ge}_{0.05}\right)_{2}$, theoretical efforts have been made [10-12]. In particular, a scenario asserting that $f$ electrons undergo a localized to itinerant transition was extensively discussed [10,11]. However, $\mathrm{YbRh}_{2} \mathrm{Si}_{2}$ shows a large Sommerfeld constant as $\sim 1.7 / \mathrm{J} \mathrm{mol} \mathrm{K}^{2}$ even inside of the antiferromagnetic (AF) phase [8], indicating that heavy quasiparticles are responsible for the AF state. Indeed, a band-structure calculation showed evidence contrary to the scenario [13] by demonstrating that a tiny valence change of $\mathrm{Yb}$ can explain the Hall-coefficient measurement in $\mathrm{YbRh}_{2} \mathrm{Si}_{2}$ [14].

Recently, in $\beta$ - $\mathrm{YbAlB}_{4}$ the valence of $\mathrm{Yb}$ has been detected as $\mathrm{Yb}^{+2.75}(0.754 f$ hole per $\mathrm{Yb})$ at $T=20 \mathrm{~K}$, suggesting strong valence fluctuations [15]. Close relation of anomalous critical phenomena and valence fluctuations has been also indicated in $\mathrm{Ce}_{0.9-x} \mathrm{Th}_{0.1} \mathrm{La}_{x}$ [16] and in $\mathrm{YbAuCu}_{4}$ [17]. Both are related to typical valence-transition materials: One is Ce metal, well known as $\gamma-\alpha$ transition [18], and the other is $\mathrm{YbInCu}_{4}$ [19], both showing a discontinuous valence jump of a $\mathrm{Ce}$ and $\mathrm{Yb}$ ion, respectively, when $T$ and $P$ are varied. Since the first-order valence transition is an isostructural transition, the critical end point exists in the $T-P$ (and chemical composition) phase diagram, as in the liquid-gas transition.

At $x \approx 0.1$ in $\mathrm{Ce}_{0.9-x} \mathrm{Th}_{0.1} \mathrm{La}_{x}$, at which the critical end point is most suppressed and is close to $T=0 \mathrm{~K}$ [16], critical phenomena rising from the quantum critical end point were revealed: $T$-linear resistivity emerges prominently and uniform spin susceptibility is enhanced at low $T$ giving rise to a large Wilson ratio, $R_{W} \sim 3$. In $\mathrm{YbAuCu}_{4}$, the uniform spin susceptibility is enhanced as $\chi(T) \sim T^{-0.6}$ as $T$ decreases in spite of the fact that the AF transition takes place at $T_{N}=0.8 \mathrm{~K}$ [20], similarly to $\mathrm{YbRh}_{2} \mathrm{Si}_{2}$. Furthermore, the sharp $\mathrm{Yb}$-valence crossover temperature $T_{v}^{*}(H)$ is induced by applying a magnetic field [17], suggesting that $\mathrm{YbAuCu}_{4}$ is located in the vicinity of the quantum critical end point of the valence transition. It should also be noted that the $T-H$ phase diagram of $\mathrm{YbAuCu}_{4}$ [17] closely resembles that of $\mathrm{YbRh}_{2} \mathrm{Si}_{2}$ [6]. The $H$ dependence of the crossover temperature $T^{*}(H)$, emerging in several physical quantities [6], whose origin is unclear in $\mathrm{YbRh}_{2} \mathrm{Si}_{2}$ is quite similar to the $T_{v}^{*}(H)$ in $\mathrm{YbAuCu}_{4}$.

These observations strongly suggest the importance of quantum criticality of valence transition as a key mechanism of unconventional critical phenomena. From this viewpoint, in this Letter, we resolve this outstanding puzzle by showing that (a) uniform spin susceptibility diverges with anomalous criticality $\chi(T) \sim T^{-\zeta}$ with $0.5 \lesssim \zeta \lesssim 0.7$ in paramagnetic metals even without proximity to a ferromagnetic phase and (b) $T$-linear resistivity emerges in the wide- $T$ range.

Let us start our discussion by introducing a minimal model which describes the essential part of the $\mathrm{Ce}-$ and $\mathrm{Yb}$-based systems in the standard notation: $H=$ $H_{c}+H_{f}+H_{\mathrm{hyb}}+H_{U_{f c}}$, where $H_{c}=\sum_{\mathbf{k} \sigma} \varepsilon_{\mathbf{k}} c_{\mathbf{k} \sigma}^{\dagger} c_{\mathbf{k} \sigma}$, 
$H_{f}=\varepsilon_{f} \sum_{i \sigma} n_{i \sigma}^{f}+U_{f f} \sum_{i=1}^{N} n_{i \uparrow}^{f} n_{i \downarrow}^{f}, \quad H_{\mathrm{hyb}}=$ $\sum_{\mathbf{k} \sigma} V_{\mathbf{k}}\left(f_{\mathbf{k} \sigma}^{\dagger} c_{\mathbf{k} \sigma}+c_{\mathbf{k} \sigma}^{\dagger} f_{\mathbf{k} \sigma}\right)$, and $H_{U_{f c}}=U_{f c} \sum_{i=1}^{N} n_{i}^{f} n_{i}^{c}$. The $U_{f c}$ term is the Coulomb repulsion between $4 f$ and conduction electrons (holes) in $\mathrm{Ce}(\mathrm{Yb})$ systems, which is considered to play an important role in the valence transition [21].

To consider correlation effects by $U_{f f}$, we employ the slave-boson large- $N$ expansion framework [22]. The Hamiltonian $H$ is generalized to the case with $N$-fold degeneracy from $\sigma=\uparrow, \downarrow$, and the slave-boson operator $b_{i}$ is introduced to eliminate the doubly-occupied state for $U_{f f} \rightarrow \infty$ under the constraint $\sum_{m} n_{i m}^{f}+N b_{i}^{\dagger} b_{i}=1$. The Lagrangian is written as $\mathcal{L}=\mathcal{L}_{0}+\mathcal{L}^{\prime}$ :

$$
\begin{aligned}
\mathcal{L}_{0}= & \sum_{\mathbf{k} m} c_{\mathbf{k} m}^{\dagger}\left(\partial_{\tau}+\bar{\varepsilon}_{\mathbf{k}}\right) c_{\mathbf{k} m}+\sum_{\mathbf{k} \mathbf{k}^{\prime} m} f_{\mathbf{k} m}^{\dagger}\left(\partial_{\tau}+\bar{\varepsilon}_{\mathbf{k}-\mathbf{k}^{\prime}}^{f}\right) f_{\mathbf{k}^{\prime} m} \\
& +\frac{V}{\sqrt{N_{s}}} \sum_{\mathbf{k} \mathbf{k}^{\prime} m}\left(c_{\mathbf{k} m}^{\dagger} f_{\mathbf{k}^{\prime} m} b_{\mathbf{k}-\mathbf{k}^{\prime}}^{\dagger}+\text { H.c. }\right)+\frac{N}{N_{s}} \sum_{\mathbf{k} \mathbf{k}^{\prime}} b_{\mathbf{k}}^{\dagger} \lambda_{\mathbf{k}-\mathbf{k}^{\prime}} b_{\mathbf{k}^{\prime}} \\
\mathcal{L}^{\prime}= & -\frac{U_{f c}}{2} \sum_{i m}\left(n_{i m}^{c}+n_{i m}^{f}\right)+\frac{U_{f c}}{N} \sum_{i m m^{\prime}} n_{i m}^{f} n_{i m^{\prime}}^{c},
\end{aligned}
$$

where $\lambda_{\mathbf{k}}$ is the Lagrange multiplier to impose the constraint, and $\bar{\varepsilon}_{\mathbf{k}} \equiv \varepsilon_{\mathbf{k}}+\frac{U_{f c}}{2}$ and $\bar{\varepsilon}_{\mathbf{k}-\mathbf{k}^{\prime}}^{f} \equiv\left(\varepsilon_{f}+\frac{U_{f c}}{2}\right) \delta_{\mathbf{k k}^{\prime}}+$ $\frac{1}{\sqrt{N_{s}}} \lambda_{\mathbf{k}-\mathbf{k}^{\prime}}$. We here separate $\mathcal{L}$ as $\mathcal{L}_{0}$ and $\mathcal{L}^{\prime}$ to perform the expansion with respect to the $U_{f c}$ term after taking account of the local correlation of the $U_{f f}$ term.

For $\exp \left(-S_{0}\right)$ with the action $S_{0}=\int_{0}^{\beta} d \tau \mathcal{L}_{0}(\tau)$, the saddle point solution is obtained via the stationary condition $\delta S_{0}=0$ by approximating spatially uniform and time independent ones, i.e., $\lambda_{\mathbf{q}}=\lambda \delta_{\mathbf{q}}$ and $b_{\mathbf{q}}=b \delta_{\mathbf{q}}$. The solution is obtained by solving mean-field equations $\partial S_{0} / \partial \lambda=0$ and $\partial S_{0} / \partial b=0$ self-consistently.

For $S^{\prime}=\int_{0}^{\beta} d \tau \mathcal{L}^{\prime}(\tau)$, we introduce the identity applied by a Stratonovich-Hubbard transformation $e^{-S^{\prime}}=$ $\int \mathcal{D} \varphi \exp \left[\sum_{i m} \int_{0}^{\beta} d \tau\left\{-\frac{U_{f c}}{2} \varphi_{i m}(\tau)^{2}+i \frac{U_{f c}}{\sqrt{N}}\left(c_{i m} f_{i m}^{\dagger}-\right.\right.\right.$ $\left.\left.\left.f_{i m} c_{i m}^{\dagger}\right)\right\} \varphi_{i m}(\tau)\right]$. The partition function is expressed as $Z=\int \mathcal{D}\left(c c^{\dagger} f f^{\dagger} \varphi\right) \exp (-S)$ with $S=S_{0}+S^{\prime}$. By performing Grassmann number integrations for $c c^{\dagger}$ and $f f^{\dagger}$, we obtain $Z=\int \mathcal{D} \varphi \exp (-S[\varphi])$ with $S[\varphi]=$ $\sum_{m} \sum_{\bar{q}} \varphi_{m}(\bar{q}) \varphi_{m}(-\bar{q})-\operatorname{Tr} \ln \left[-\hat{G}_{0}^{-1}+\hat{V}\right]-\frac{\beta N}{\sqrt{N_{s}}} \lambda|b|^{2}+$ $\beta N q_{0} \sqrt{N_{s}} \lambda$. Here, the abbreviation $\bar{q} \equiv\left(\mathbf{q}, i \omega_{l}\right)$ with $\omega_{l}=2 l \pi T$ is used, and $\hat{G}_{0}$ and $\hat{V}$ are defined as

$$
\hat{G}_{0}^{-1} \equiv\left(\begin{array}{cc}
i \varepsilon_{n}-\bar{\varepsilon}_{\mathbf{k}} & -\frac{V b^{*}}{\sqrt{N_{s}}} \\
-\frac{V b}{\sqrt{N_{s}}} & i \varepsilon_{n}-\bar{\varepsilon}_{\mathbf{0}}^{f}
\end{array}\right) \delta_{\mathbf{k} \mathbf{k}^{\prime},} \quad \hat{V} \equiv\left(\begin{array}{cc}
0 & \tilde{\varphi}_{m \bar{k} \bar{k}^{\prime}} \\
\tilde{\varphi}_{m \bar{k} \bar{k}^{\prime}} & 0
\end{array}\right)
$$

with $\tilde{\varphi}_{m \bar{k} \bar{k}^{\prime}} \equiv \frac{U_{f c}}{\sqrt{\beta N N_{s}}} \varphi_{m}\left(\bar{k}-\bar{k}^{\prime}\right)$, respectively. Here, $\bar{k} \equiv$ $\left(\mathbf{k}, i \varepsilon_{n}\right)$ with $\varepsilon_{n}=(2 n+1) \pi T$, and each matrix element of $\hat{G}_{0}$ is defined as $\left(\hat{G}_{0}\right)_{11} \equiv G_{0}^{c c},\left(\hat{G}_{0}\right)_{12} \equiv G_{0}^{c f},\left(\hat{G}_{0}\right)_{21} \equiv G_{0}^{f c}$, and $\quad\left(\hat{G}_{0}\right)_{22} \equiv G_{0}^{f f} . \quad$ By $\quad$ using $\quad \operatorname{Tr} \ln \left[-\hat{G}_{0}^{-1}+\hat{V}\right]=$ $\operatorname{Tr} \ln \left[-\hat{G}_{0}^{-1}\right]-\sum_{n=1}^{\infty} \frac{1}{n} \operatorname{Tr}\left(\hat{G}_{0} \hat{V}\right)^{n}$, we obtain

$$
\begin{aligned}
S[\varphi]= & \sum_{m}\left[\frac{1}{2} \sum_{\bar{q}} \Omega_{2}(\bar{q}) \varphi_{m}(\bar{q}) \varphi_{m}(-\bar{q})+\sum_{\bar{q}_{1}, \bar{q}_{2}, \bar{q}_{3}} \Omega_{3}\left(\bar{q}_{1}, \bar{q}_{2}, \bar{q}_{3}\right)\right. \\
& \times \varphi_{m}\left(\bar{q}_{1}\right) \varphi_{m}\left(\bar{q}_{2}\right) \varphi_{m}\left(\bar{q}_{3}\right) \delta\left(\sum_{i=1}^{3} \bar{q}_{i}\right) \\
& +\sum_{\bar{q}_{1}, \bar{q}_{2}, \bar{q}_{3}, \bar{q}_{4}} \Omega_{4}\left(\bar{q}_{1}, \bar{q}_{2}, \bar{q}_{3}, \bar{q}_{4}\right) \times \varphi_{m}\left(\bar{q}_{1}\right) \varphi_{m}\left(\bar{q}_{2}\right) \\
& \left.\times \varphi_{m}\left(\bar{q}_{3}\right) \varphi_{m}\left(\bar{q}_{4}\right) \delta\left(\sum_{i=1}^{4} \bar{q}_{i}\right)+\cdots\right] .
\end{aligned}
$$

Here, constant terms independent of $\varphi_{m}(\bar{q})$ are omitted in Eq. (2) since they merely shift the origin of the free energy of the system. The coefficient of the quadratic term is given by

$$
\Omega_{2}\left(\mathbf{q}, i \omega_{l}\right)=U_{f c}\left[1-\frac{2 U_{f c}}{N}\left\{\chi_{0}^{f f c c}\left(\mathbf{q}, i \omega_{l}\right)-\chi_{0}^{c f c f}\left(\mathbf{q}, i \omega_{l}\right)\right\}\right],
$$

where $\quad \chi_{0}^{\alpha \beta \gamma \delta}\left(\mathbf{q}, i \omega_{l}\right) \equiv-\frac{T}{N_{\mathrm{s}}} \sum_{\mathbf{k}, n} G_{0}^{\alpha \beta}\left(\mathbf{k}+\mathbf{q}, i \varepsilon_{n}+i \omega_{l}\right)$ $G_{0}^{\gamma \delta}\left(\mathbf{k}, i \varepsilon_{n}\right)$. Since long wavelength $|\mathbf{q}| \ll q_{c}$ around $\mathbf{q}=\mathbf{0}$ and low frequency $|\omega| \ll \omega_{c}$ regions play dominant roles in critical phenomena with $q_{c}$ and $\omega_{c}$ being cutoffs for momentum and frequency in the order of inverse of the lattice constant and the effective Fermi energy, respectively, $\Omega_{i}$ for $i=2,3$, and 4 are expanded for $q$ and $\omega$ around $(\mathbf{0}, 0)$ :

$$
\Omega_{2}\left(\mathbf{q}, i \omega_{l}\right) \approx \eta+A q^{2}+C \frac{\left|\omega_{l}\right|}{q}
$$

where $\eta=U_{f c}\left[1-\frac{2 U_{f c}}{N}\left\{\chi_{0}^{f f c c}(\mathbf{0}, 0)-\chi_{0}^{c f c f}(\mathbf{0}, 0)\right\}\right]$, $\Omega_{3}\left(q_{1}, q_{2}, q_{3}\right) \approx v_{3} / \sqrt{\beta N_{s}}, \quad$ and $\Omega_{4}\left(q_{1}, q_{2}, q_{3}, q_{4}\right) \approx$ $v_{4} /\left(\beta N_{s}\right)$.

Different from ordinary critical phenomena of spin fluctuations [1-4], there appears a cubic term in Eq. (2) in general for the valence fluctuation case [23]. Let us here apply the Hertz's renormalization-group procedure [3] to $S[\varphi]$ : (a) Integrating out high momentum and frequency parts for $q_{c} / s<q<q_{c}$ and $\omega_{c} / s^{z}<\omega<\omega_{c}$, respectively, with $s$ being a dimensionless scaling parameter $(s \geq 1)$ and $z$ the dynamical exponent. (b) Scaling of $q$ and $\omega$ by $q^{\prime}=s q$ and $\omega^{\prime}=s^{z} \omega$. (c) Rescaling of $\varphi$ by $\varphi^{\prime}\left(\mathbf{q}^{\prime}, \omega^{\prime}\right)=s^{a} \varphi\left(\mathbf{q}^{\prime} / s, \omega^{\prime} / s\right)$. Then, we determined that to make the Gaussian term in Eq. (2) scale invariant, $a$ must satisfy $a=-(d+z+2) / 2$ with $d$ spatial dimension and the dynamical exponent $z=3$. The renormalization-group equations for coupling constants $v_{j}$ are derived as $\frac{d v_{3}}{d s}=$ $[6-(d+z)] v_{3}+O\left(v_{3}^{2}\right)$, and $\frac{d v_{4}}{d s}=[4-(d+z)] v_{4}+$ $O\left(v_{4}^{2}\right)$, for cubic and quadratic terms, respectively. By solving these equations, it is shown that higher order terms than the Gaussian term are irrelevant

$$
\lim _{s \rightarrow \infty} v_{j}(s)=0 \text { for } j \geq 3
$$


for $d+z>6$. For the case of $d=3$ and $z=3$, it is shown that the cubic term is marginally irrelevant [21]. Hence, the universality class of the criticality of valence fluctuations belongs to the Gaussian fixed point. This implies that critical valence fluctuations are qualitatively described by the RPA framework with respect to $U_{f c}$. The coefficient of the Gaussian term in Eq. (2) is nothing but the inverse of the valence susceptibility $\Omega_{2}\left(\mathbf{q}, i \omega_{l}\right) \equiv \chi_{v}\left(\mathbf{q}, i \omega_{l}\right)^{-1}$. Since evaluation of $\chi_{0}^{f f c c}\left(\mathbf{q}, i \omega_{l}\right)$ and $\chi_{0}^{c f c f}\left(\mathbf{q}, i \omega_{l}\right)$ using the saddle point solution for $\exp \left(-S_{0}\right)$ concludes $\chi_{0}^{f f c c} \gg \chi_{0}^{c f c f}$ (see Fig. 2 and text below), it turns out that $\chi_{v}$ is expressed by the RPA form $\chi_{v}\left(\mathbf{q}, i \omega_{l}\right)=$ $\int_{0}^{\beta} d \tau\left\langle T_{\tau} n_{f}(\mathbf{q}, \tau) n_{f}(-\mathbf{q}, 0)\right\rangle e^{i \omega_{l} \tau} \approx U_{f c}^{-1}\left[1-\frac{2 U_{f c}}{N} \chi_{0}^{f f c c}\right.$ $\left.\left(\mathbf{q}, i \omega_{l}\right)\right]^{-1}$, as shown in Fig. 1.

An important consequence of this result is that dynamical $f$-spin susceptibility $\chi_{f}^{+-}\left(\mathbf{q}, i \omega_{l}\right) \equiv \int_{0}^{\beta} d \tau\left\langle T_{\tau} S_{f}^{+}(\mathbf{q}, \tau)\right.$ $\left.S_{f}^{-}(-\mathbf{q}, 0)\right\rangle e^{i \omega_{l} \tau}$ has a common structure with $\chi_{v}$ in the RPA framework as shown in Fig. 1. At the quantum critical end point of the valence transition, namely, the quantum critical point $(\mathrm{QCP})$, the valence susceptibility $\chi_{v}(\mathbf{0}, 0)$ diverges. The common structure indicates that $\chi_{f}^{+-}(\mathbf{0}, 0)$ also diverges at the QCP. The uniform spin susceptibility is given by $\chi \approx \chi_{s}^{f} \approx \frac{3}{2} \mu_{B}^{2} g_{f}^{2} \chi_{f}^{+-}(\mathbf{0}, 0)$ with $\chi_{s}^{f}$ uniform $f$-spin susceptibility, $\mu_{B}$ the Bohr magneton, and $g_{f}$ Lande's $g$ factor for $f$ electrons. This gives a qualitative explanation for the fact that uniform spin susceptibility diverges at the $\mathrm{QCP}$ of valence transition under a magnetic field, which was shown by the slave-boson mean-field theory applied to the Hamiltonian $H$ [24]. Numerical calculations for $H$ in $d=1$ by the density matrix renormalization group [24] and in $d=\infty$ by the dynamical mean field theory [25] also showed the simultaneous divergence of $\chi_{v}$ and uniform spin susceptibility under the magnetic field, reinforcing the above argument based on RPA.

The other important point of the present theory is that the "unperturbed" term $\mathcal{L}_{0}$, i.e., $\hat{G}_{0}$, already contains the local correlation effect by $U_{f f}$. This effect plays a key role in critical phenomena in $\mathrm{Ce}$ - and $\mathrm{Yb}$-based systems, which will be shown below to be the origin of the unconventional criticality. The local correlation effect emerges as dispersionless, almost $q$-independent $\chi_{0}^{f f c c}(\mathbf{q}, 0)$ and $\chi_{0}^{c f c f}(\mathbf{q}, 0)$ in Eq. (3), as shown in Fig. 2(a). Here, the saddle point solution for $\exp \left(-S_{0}\right)$ is employed for a typical parameter set of heavy-electron systems: $D=1$, $V=0.5$, and $U_{f f}=\infty$ at total filling $n=7 / 8$ with

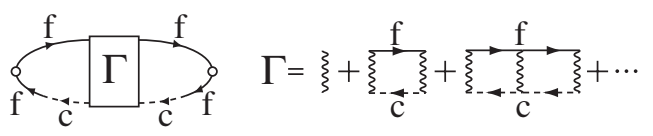

FIG. 1. Feynman diagrams for dynamical valence susceptibility and dynamical spin susceptibility for $f$ electrons. Solid lines and dashed lines represent the $f$ - and conduction-electron Green functions, $G_{0}^{f f}$ and $G_{0}^{c c}$, respectively. Wiggly lines represent $U_{f c}$. $\varepsilon_{\mathbf{k}}=\mathbf{k}^{2} /\left(2 m_{0}\right)-D$ and $n \equiv \bar{n}_{f}+\bar{n}_{c}$, where $\bar{n}_{f}$ and $\bar{n}_{c}$ are the number of $f$ electrons and conduction electrons per "spin" and site, respectively. The bare mass $m_{0}$ is chosen such that the integration from $-D$ to $D$ of the density of states of conduction electrons per "spin" is equal to 1 . This local nature is reflected in the inverse of valence susceptibility in Eq. (4) as an extremely small coefficient $A$. We note here that this flat $-q$ result is obtained not only for deep $\varepsilon_{f}$ with $\bar{n}_{f}=1 / 2$ in the Kondo regime, but also for shallow $\varepsilon_{f}$ with $\bar{n}_{f}<1 / 2$ in the valence-fluctuating regime [see Fig. 2(b)]. Here, we note that the $c-f$ hybridization is always finite.

To clarify how this local nature causes unconventional criticality, we construct a self-consistent renormalization (SCR) theory for valance fluctuations. Although higher order terms $v_{j}(j \geq 3)$ in $S[\varphi]$ are irrelevant as shown in Eq. (5), the effect of their mode couplings affects low- $T$ physical quantities significantly as is well known in spinfluctuation theories [1-4]. To construct the action using the best Gaussian taking account of the mode-coupling effects up to the 4th order $(j \leq 4)$ in $S[\varphi]$, we employ Feynman's inequality on the free energy: $F \leq F_{\text {eff }}+T\left\langle S-S_{\text {eff }}\right\rangle_{\text {eff }} \equiv$ $\tilde{F}(\eta)$. Here, $S_{\text {eff }}[\varphi]=\frac{1}{2} \sum_{m} \sum_{\mathbf{q}, l}\left(\eta+A q^{2}+C_{q}\left|\omega_{l}\right|\right)$ $\left|\varphi_{m}\left(\mathbf{q}, i \omega_{l}\right)\right|^{2}$, and $\eta$ is determined to make $\tilde{F}(\eta)$ be optimum. By optimal condition $d \tilde{F}(\eta) / d \eta=0$, the self-consistent equation for $\eta$, i.e., the SCR equation, is obtained: $\eta=\eta_{0}+3 v_{4}\left\langle\varphi_{m}^{2}\right\rangle_{\text {eff }}^{2} / N_{s}$, where $\left\langle\varphi_{m}^{2}\right\rangle_{\text {eff }}=$ $T \sum_{\mathbf{q}, l}\left(\eta+A q^{2}+C_{q}\left|\omega_{l}\right|\right)^{-1}$. Here, we write $\left\langle\varphi_{m}^{2}\right\rangle_{\mathrm{eff}}$ in a general form using $C_{q}$, which is given by $C_{q} \equiv$ $C / \max \left\{q, l_{i}^{-1}\right\}$ with $l_{i}$ being the mean-free path by impurity scattering [26]. When the system is clean, i.e., $C_{q}=C / q$, the SCR equation in $d=3$ in the $A q_{B}^{2} \lesssim \eta$ regime with $q_{B}$ being the momentum at the Brillouin Zone is given by

$$
y=y_{0}+\frac{3}{2} y_{1} t\left[\frac{x_{c}^{3}}{6 y}-\frac{1}{2 y} \int_{0}^{x_{c}} d x \frac{x^{3}}{x+\frac{t}{6 y}}\right]
$$

where $y \equiv \eta /\left(A q_{B}^{2}\right), t \equiv T / T_{0}, T_{0} \equiv A q_{B}^{3} /(2 \pi C), x \equiv$ $q / q_{B}, x_{c} \equiv q_{c} / q_{B}$, and $y_{0}$ and $y_{1}$ are constants. When $y \gg t, y \propto t^{2 / 3}$ is obtained from Eq. (6) at the QCP with $y_{0}=0$. This indicates that the valence susceptibility
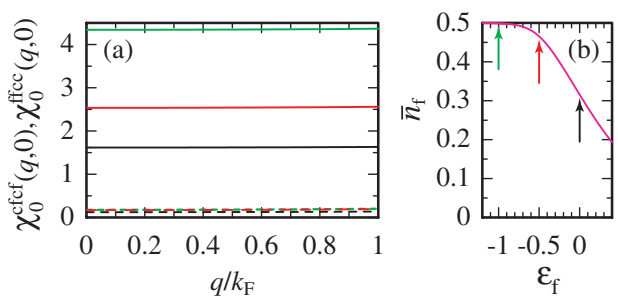

FIG. 2 (color). (a) $q$ dependence of $\chi_{0}^{f f c c}(q, 0)$ (solid line) and $\chi_{0}^{c f c f}(q, 0)$ (dashed line) calculated by using saddle point solution of $\exp \left(-S_{0}\right)$ for $\varepsilon_{f}=-1$ (green), -0.5 (red), and 0.0 (black). (b) $\bar{n}_{f}$ vs $\varepsilon_{f}$. (a) and (b) are results for $D=1, V=$ 0.5 , and $U_{f f}=\infty$ at $n=7 / 8$. 

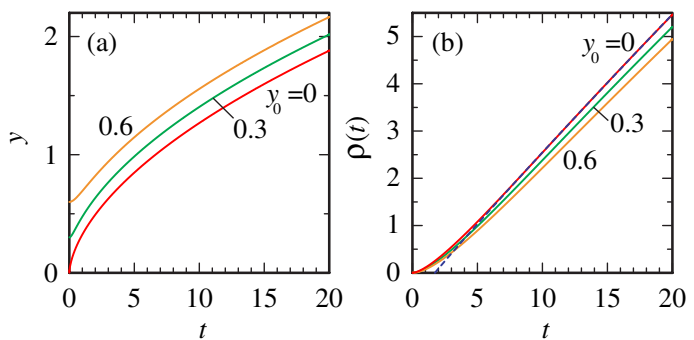

FIG. 3 (color online). (a) Numerical solutions of Eq. (6) for $y_{0}=0.0$ (at QCP), 0.3, and 0.6 at $y_{1}=1$ and $x_{c}=1$. (b) Electrical resistivity $\rho(T)$ calculated by using $y(t)$ in (a). Dashed line represents the linear- $t$ fit.

shows unconventional criticality $\chi_{v}(\mathbf{0}, 0)=\eta^{-1} \propto t^{-2 / 3}$. Figure 3(a) shows numerical solutions of Eq. (6). Note here that the coefficient $A$ is quite small as shown above, giving rise to quite small $T_{0}\left(=A q_{B}^{3} /(2 \pi C) \ll T_{F}\right)$ so that the region of $t \equiv T / T_{0}$ shown in Fig. 3 corresponds to that of $T \ll T_{F} \sim O(D)$. Hence, a wide range of $t=T / T_{0}$ is shown in the plot. The least square fit of the data for $5 \leq t \leq 100$ gives $y \propto t^{0.551}$. Since the Gaussian fixed point ensures the simultaneous divergence of valence susceptibility and uniform $f$-spin susceptibility as discussed above, $\chi_{s}^{f}$ shows divergent behavior $\sim t^{-\zeta}$ with $0.5 \lessgtr \zeta \lesssim$ 0.7 depending on the temperature range in agreement with experiments in $\mathrm{YbRh}_{2}\left(\mathrm{Si}_{0.95} \mathrm{Ge}_{0.05}\right)_{2}$ and $\beta$ - $\mathrm{YbAlB}_{4}$. We note that the NMR or NQR relaxation rate is shown to be $\left(T_{1} T\right)^{-1} \sim \chi_{s}^{f}(t) \propto t^{-\zeta}$, which also quantitatively agrees with $\left(T_{1} T\right)^{-1} \sim T^{-0.5}$ in $\mathrm{YbRh}_{2} \mathrm{Si}_{2}$ [27].

We should note here that in the $T \rightarrow 0$ limit, although it may be experimentally difficult to access such a low temperature overcoming the smallness of $A$, the SCR equation follows the conventional $z=3$ type $[1,3,4]$ in the clean system, giving rise to $y \propto t^{4 / 3}$. Then, at the QCP $\left(y_{0}=0\right)$, as $t$ decreases, a crossover from $y \propto t^{2 / 3}$ to $y \propto t^{4 / 3}$ occurs. In reality, however, because of the smallness of $A$, the low- $T$ range is extremely elongated by the relation $t=T / T_{0}$ with $T_{0} \propto A$, which makes it possible that unconventional criticality dominates over the experimentally accessible low-temperature region.

We note that the electrical resistivity $\rho(T)$ shows a $T$-linear dependence in the regime $t \geq 5 \quad(y \geq 1)$ where Eq. (6) is applicable, as shown in Fig. 3(b). Here, following a formalism of Ref. [28], $\rho(T)$ is calculated as $\rho(T) \propto \frac{1}{T} \int_{-\infty}^{\infty} d \omega \omega n(\omega)[n(\omega)+1] \int_{0}^{q_{c}} d q q^{3} \operatorname{Im} \chi_{v}^{R}(q, \omega)$ with $n(\omega)=1 /\left(e^{\beta \omega}-1\right)$ being the Bose distribution function, and $\chi_{v}^{R}(q, \omega)=\left(\eta+A q^{2}-i C_{q} \omega\right)^{-1}$, a retarded valence susceptibility. Here, $y(t)$ in Fig. 3(a) is used for the clean system $C_{q}=C / q$, and the normalization constant is taken as 1 in the $\rho(t)$ plot. The emergence of $\rho(t) \propto t$ behavior can be understood from the locality of valence fluctuations: In the system with a small coefficient $A$, where the local character is strong, the dynamical exponent may be regarded as $z=\infty$ when we write $C_{q}$ in a general form as $C_{q}=C / q^{z-2}$. By using this expression in $\chi_{v}^{R}(q, \omega)$ in the calculation of $\rho(T)$ for $z=\infty$, we obtain $\rho(T) \propto T$ toward $T \rightarrow 0 \mathrm{~K}$. This result indicates that the locality of valence fluctuations causes the $T$-linear resistivity. The emergence of $\rho(T) \propto T$ by valence fluctuations was shown theoretically on the basis of the valence susceptibility $\chi_{v}$ which has an approximated form for $z=\infty$ in Ref. [29].

The evaluation of the quasiparticle self-energy for a valence fluctuation exchange process by using the $\chi_{v}$ shows that $\operatorname{Re} \Sigma(\varepsilon) \propto \varepsilon \ln (\varepsilon)$ [30], which leads to a logarithmic- $T$ dependence in the specific heat $C / T$ for a certain- $T$ range [31]. The detailed $T$ dependence of $C / T$ will be discussed in a separate paper.

[1] T. Moriya, Spin Fluctuations in Itinerant Electron Magnetism (Springer-Verlag, Berlin, 1985).

[2] T. Moriya et al., J. Phys. Soc. Jpn. 64, 960 (1995).

[3] J. A. Hertz, Phys. Rev. B 14, 1165 (1976).

[4] A. J. Millis, Phys. Rev. B 48, 7183 (1993).

[5] O. Trovarelli et al., Phys. Rev. Lett. 85, 626 (2000).

[6] P. Gegenwart et al., Science 315, 969 (2007).

[7] P. Gegenwart et al., Phys. Rev. Lett. 94, 076402 (2005).

[8] J. Custers et al., Nature (London) 424, 524 (2003).

[9] S. Nakatsuji et al., Nature Phys. 4, 603 (2008); Y. Matsumoto et al., Phys. Status Solidi B 247, 720 (2010).

[10] Q. Si et al., Nature (London) 413, 804 (2001).

[11] P. Coleman et al., J. Phys. Condens. Matter 13, R723 (2001).

[12] T. Misawa et al., J. Phys. Soc. Jpn. 77, 093712 (2008).

[13] M. R. Norman, Phys. Rev. B 71, 220405 (2005).

[14] S. Paschen et al., Nature (London) 432, 881 (2004).

[15] M. Okawa et al., Phys. Rev. Lett. 104, 247201 (2010).

[16] J. C. Lashley et al., Phys. Rev. Lett. 97, 235701 (2006).

[17] S. Wada et al., J. Phys. Condens. Matter 20, 175201 (2008).

[18] K. A. Gschneidner and L. Eyring, Handbook on the Physics and Chemistry of Rare Earths (North-Holland, Amsterdam, 1978).

[19] I. Felner and I. Nowik, Phys. Rev. B 33, 617 (1986).

[20] J. L. Sarrao et al., Phys. Rev. B 59, 6855 (1999).

[21] K. Miyake, J. Phys. Condens. Matter 19, 125201 (2007).

[22] Y. Onishi et al., J. Phys. Soc. Jpn. 69, 3955 (2000).

[23] There exists the region where the cubic term in Eq. (2) vanishes in the $\varepsilon_{f}-U_{f c}$ plane for each $V$, making the higher order terms $v_{j}(j \geq 3)$ irrelevant for $d+z>4$.

[24] S. Watanabe et al., Phys. Rev. Lett. 100, 236401 (2008).

[25] T. Sugibayashi et al., Physica C (Amsterdam) (to be published).

[26] K. Miyake et al., Physica B (Amsterdam) 259, 676 (1999).

[27] K. Ishida et al., Phys. Rev. Lett. 89, 107202 (2002).

[28] K. Ueda et al., J. Phys. Soc. Jpn. 39, 605 (1975).

[29] A. T. Holmes, D. Jaccard, and K. Miyake, Phys. Rev. B 69, 024508 (2004).

[30] C. M. Varma, Phys. Rev. Lett. 75, 898 (1995).

[31] S. Watanabe et al., arXiv:0906.3986. 